\title{
SOME PARTICULAR ENTRIES OF THE TWO-PARAMETER KOSTKA MATRIX
}

\author{
JOHN R. STEMBRIDGE
}

(Communicated by Jeffry N. Kahn)

\begin{abstract}
Macdonald has defined a two-parameter refinement of the Kostka matrix, denoted $K_{\lambda, \mu}(q, t)$. The entries are rational functions of $q$ and $t$, but he has conjectured that they are in fact polynomials with nonnegative integer coefficients. We prove two results that support this conjecture. First, we prove that if $\mu$ is a partition with at most two columns (or at most two rows), then $K_{\lambda, \mu}(q, t)$ is indeed a polynomial. Second, we provide a combinatorial interpretation of $K_{\lambda, \mu}(q, t)$ for the case in which $\mu$ is a hook. This interpretation proves in this case that not only are the entries polynomials, but also that their coefficients are nonnegative integers.
\end{abstract}

\section{INTRODUCTION}

Macdonald has defined a basis $P_{\lambda}(q, t)$ of the ring of symmetric functions depending on two parameters $q$ and $t$ [M2]. Numerous important families of symmetric functions are limiting or special cases of the $P_{\lambda}(q, t)$ 's, including Schur functions, zonal polynomials, Jack polynomials, and Hall-Littlewood symmetric functions. Although these new symmetric functions possess many properties similar to those of the more familiar special cases, these properties tend to be much harder to prove, owing to the lack of (quoting [M2]) "any sort of usable 'closed formula' " for $P_{\lambda}(q, t)$.

The coefficients of the $P_{\lambda}(q, t)$ 's are (complicated) rational functions of $q$ and $t$. Macdonald has also introduced certain renormalizations of the $P_{\lambda}$ 's, denoted $J_{\lambda}(q, t)$, that he conjectures to have polynomial coefficients (i.e., coefficients in $\mathbf{Z}[q, t])$. Moreover, there is a transition matrix, denoted $K_{\lambda, \mu}(q, t)$, between the $J_{\mu}$ 's and another basis $S_{\lambda}$ that generalizes in a natural way the Kostka matrix $K_{\lambda, \mu}$. The entries of this two-parameter matrix are conjectured by Macdonald to be polynomials with nonnegative integer coefficients. Neither the fact that the entries are polynomials, nor that their coefficients are nonnegative has been proved in general. Recently, Garsia and Haiman [GH] have constructed a family of finite-dimensional bigraded $S_{n}$-modules $R_{\mu}$ which they conjecture to have the property that for each partition $\lambda$, the Poincare series of multiplicities for the isotypic component of type $\lambda$ in $R_{\mu}$ is $K_{\lambda, \mu}(q, t)$. They

Received by the editors August 12, 1992 and, in revised form, September 24, 1992.

1991 Mathematics Subject Classification. Primary 05 E05.

Partially supported by NSF Grants DMS-9057192 and DMS-9101898. 
have also announced (in [GH]) a proof of this evidently stronger conjecture for the case in which $\mu$ has two rows or when $\mu$ is a hook.

The purpose of this paper is to give short, elementary proofs of the following:

(1) An explicit formula for $K_{\lambda, \mu}(q, t)$ for partitions $\mu$ with at most two columns or, equivalently (by duality), at most two rows.

(2) A combinatorial interpretation of $K_{\lambda, \mu}(q, t)$ for the case in which $\mu$ is a hook.

The formula in (1) is expressed in terms of the Kostka-Foulkes polynomials $K_{\lambda, \nu}(t)$. It shows, in particular, that $K_{\lambda, \mu}(q, t)$ is indeed a polynomial for the cases cited, but it does not show that the coefficients are nonnegative. On the other hand, the combinatorial interpretation in (2) does explicitly demonstrate the nonnegativity of the coefficients and thus proves Macdonald's conjecture for the cases it covers. Further special cases (for example, the case in which $\lambda$ is a hook) and other supporting evidence for the conjecture can be found in [M2, $\S 6]$.

We will assume familiarity with the notation of [M1, M2]. This includes, for example, the definitions of the previously mentioned symmetric functions $P_{\lambda}(q, t)$ and $J_{\lambda}(q, t)$, the Schur functions $s_{\lambda}$, the modified Schur functions $S_{\lambda}$, the Hall-Littlewood functions $Q_{\lambda}(t)$, and the associated Kostka-Foulkes polynomials $K_{\lambda, \nu}(t)$.

\section{THE CASE OF TWO COLUMNS}

For integers $n \geq 0$ and indeterminates $a$ and $t$, define

$$
(a ; t)_{n}=(1-a)(1-a t) \cdots\left(1-a t^{n-1}\right) .
$$

Let $\left[\begin{array}{l}n \\ k\end{array}\right]_{t}=\left(t^{n} ; t^{-1}\right)_{k} /\left(t^{k} ; t^{-1}\right)_{k}$ denote the Gaussian polynomial.

Theorem 1.1. We have

$$
K_{\lambda, 2^{r} 1^{n-2 r}}(q, t)=\sum_{s=0}^{r} q^{r-s}\left(t^{n-r} q ; t^{-1}\right)_{s}\left[\begin{array}{l}
r \\
s
\end{array}\right]_{t} K_{\lambda, 2^{s} 1^{n-2 s}}(t) .
$$

Proof. By definition, $K_{\lambda, \mu}(q, t)$ is the transition matrix between the symmetric functions $J_{\mu}(q, t)$ and $S_{\lambda}$; i.e.,

$$
J_{\mu}(q, t)=\sum_{\lambda} K_{\lambda, \mu}(q, t) S_{\lambda} .
$$

Let us similarly define $a_{\lambda, \mu}(q, t)$ to be the transition matrix between $J_{\mu}(q, t)$ and the Hall-Littlewood functions $Q_{\lambda}(t)$, so that

$$
J_{\mu}(q, t)=\sum_{\lambda} a_{\lambda, \mu}(q, t) Q_{\lambda}(t)
$$

Recall that the transition matrix between the $J_{\mu}$ 's and the monomial symmetric functions is (by definition) lower triangular with respect to the dominance order, and the same is known to be true for the Hall-Littlewood functions $Q_{\lambda}$. Therefore, $a_{\lambda, \mu}$ must be upper triangular; i.e., $a_{\lambda, \mu}=0$ unless $\lambda \leq \mu$ in dominance order. by

Let us note that the Kostka-Foulkes matrix $K_{\lambda, \mu}(t)[\mathrm{M} 1, \mathrm{III}]$ can be defined

$$
Q_{\mu}(t)=\sum_{\lambda} K_{\lambda, \mu}(t) S_{\lambda}
$$


Since $J_{\mu}(0, t)=Q_{\mu}(t)[\mathrm{M} 2,(6.4)]$, one knows that $K_{\lambda, \mu}(t)=K_{\lambda, \mu}(0, t)$. Combining (1.1)-(1.3), we obtain

$$
K_{\lambda, \mu}(q, t)=\sum_{\nu} K_{\lambda, \nu}(t) a_{\nu, \mu}(q, t)
$$

For nonnegative integers $r, s \leq n / 2$, let us define

$$
A_{s, r}(q, t)=a_{2^{s} 1^{n-2 s}, 2^{r} 1^{n-2 r}}(q, t) .
$$

This is a slight abuse of notation, since $A_{s, r}$ also depends on $n$. In view of (1.4), it suffices to prove that

$$
A_{s, r}(q, t)=q^{r-s}\left(t^{n-r} q ; t^{-1}\right)_{s}\left[\begin{array}{l}
r \\
s
\end{array}\right]_{t} .
$$

For this, let $\varepsilon_{a, t}$ denote the specialization homomorphism defined on the ring of symmetric functions by setting $\varepsilon_{a, t}\left(p_{r}\right)=\left(1-a^{r}\right) /\left(1-t^{r}\right)$. By a result of Macdonald [M2, (5.3)], one knows that

$$
\varepsilon_{a, t} J_{\mu}(q, t)=\prod_{(i, j) \in \lambda}\left(t^{i-1}-q^{j-1} a\right)
$$

where the product ranges over all cells of the Young diagram of $\lambda$ (i.e., $1 \leq$ $\left.j \leq \lambda_{i}\right)$. In the special case $\mu=2^{r} 1^{n-2 r}$, this becomes

$$
\varepsilon_{a, t} J_{\mu}(q, t)=\prod_{i=1}^{n-r}\left(t^{i-1}-a\right) \prod_{i=1}^{r}\left(t^{i-1}-q a\right) .
$$

Similarly, since $Q_{\lambda}(t)=J_{\lambda}(0, t)$, it follows that

$$
\varepsilon_{a, t} Q_{\mu}(t)=t^{\left(\begin{array}{r}
r \\
2
\end{array}\right)} \prod_{i=1}^{n-r}\left(t^{i-1}-a\right)
$$

again under the assumption that $\mu=2^{r} 1^{n-2 r}$.

Thus, by (1.2), we have

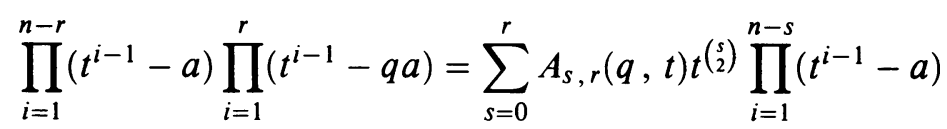

or, equivalently,

$$
\prod_{i=1}^{r}\left(t^{i-1}-q a\right)=\sum_{s=0}^{r} A_{s, r}(q, t) t^{\left(\begin{array}{c}
s \\
2
\end{array}\right)} \prod_{i=1}^{r-s}\left(t^{n-r+i-1}-a\right) .
$$

In this form, it is clear that $A_{s, r}$ is a transition matrix between two bases of the polynomial ring $F[a]$, where $F=\mathbf{Q}(q, t)$. In particular, these constraints uniquely determine $A_{s, r}$, so it suffices to prove that

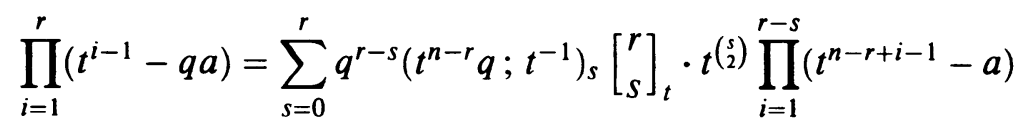


or, equivalently,

$$
b^{r}(1 / b ; t)_{r}=\sum_{s=0}^{r} z^{s}(1 / z ; t)_{s} \cdot b^{r-s}(z / b ; t)_{r-s}\left[\begin{array}{l}
r \\
s
\end{array}\right]_{t},
$$

where $b=a q$ and $z=t^{n-r} q$.

In terms of the formal power series $B(a, t ; y)=\sum_{r \geq 0}(a ; t)_{r} y^{r} /(t ; t)_{r}$, we see that (1.7) can be rewritten in the form

$$
B(1 / b, t ; b y)=B(1 / z, t ; z y) \cdot B(z / b, t ; b y) .
$$

However, by the $q$-binomial theorem (e.g., [A, (2.2.1)]), one knows that

$$
B(a, t ; y)=\frac{(a y ; t)_{\infty}}{(y ; t)_{\infty}}
$$

so (1.7) amounts to the assertion that

$$
\frac{(y ; t)_{\infty}}{(b y ; t)_{\infty}}=\frac{(y ; t)_{\infty}}{(z y ; t)_{\infty}} \cdot \frac{(z y ; t)_{\infty}}{(b y ; t)_{\infty}}
$$

Using the fact that $K_{\lambda, \mu}(q, t)=K_{\lambda^{\prime}, \mu^{\prime}}(t, q)$ [M2, §6], one may deduce from this a similar formula for $K_{\lambda, \mu}(q, t)$ for partitions $\mu$ with at most two parts.

Corollary 1.2. We have

$$
K_{\lambda,(n-r, r)}(q, t)=\sum_{s=0}^{r} t^{r-s}\left(q^{n-r} t ; q^{-1}\right)_{s}\left[\begin{array}{l}
r \\
s
\end{array}\right]_{q} K_{\lambda^{\prime}, 2^{s} 1^{n-2 s}}(q) .
$$

Remark 1.3. Since $K_{\lambda, \mu}(t)$ is lower triangular and $a_{\lambda, \mu}(q, t)$ is upper triangular, it follows that (1.4) is the LU factorization of the $(q, t)$-Kostka matrix; it is unique up to diagonal transformations. Furthermore, since $K_{\lambda, \mu}(t)$ is invertible over $\mathbf{Z}[t]$ (it has a unit diagonal), it follows that $K_{\lambda, \mu}(q, t) \in \mathbf{Z}[q, t]$ if and only if $a_{\nu, \mu}(q, t) \in \mathbf{Z}[q, t]$ for all $\nu \leq \mu$.

Remark 1.4. Let $h_{\mu}(q, t)$ denote the coefficient of the monomial symmetric function $m_{\mu}$ in $J_{\mu}(q, t)$ (i.e., the "leading term" of $\left.J_{\mu}\right)$. Since $Q_{\mu}(t)=$ $J_{\mu}(0, t)$, it follows that $h_{\mu}(0, t)$ is the coefficient of $m_{\mu}$ in $Q_{\mu}(t)$, and hence, $a_{\mu, \mu}(q, t)=h_{\mu}(q, t) / h_{\mu}(0, t)$. Using Macdonald's formula for $h_{\mu}(q, t)$ [M2, (6.1)], it follows that

$$
a_{\mu, \mu}(q, t)=\prod_{x \in \mu: a(x) \neq 0}\left(1-q^{a(x)} t^{l(x)+1}\right),
$$

where $a(x)$ and $l(x)$ denote the arm length and leg length of the hook of $\mu$ indexed by $x$.

Remark 1.5. By (1.2) and (1.5), we obtain the formula

$$
J_{2^{r} 1^{n-2 r}}(q, t)=\sum_{s=0}^{r} q^{r-s}\left(t^{n-r} q ; t^{-1}\right)_{s}\left[\begin{array}{l}
r \\
s
\end{array}\right]_{t} Q_{2^{s} 1^{n-2 s}}(t) .
$$

By means of duality [M2, (3.5)], one can also use this to give a formula for $J_{(n-r, r)}(q, t)$. It is not identical to the one given by Jing and Józefiak [JJ], since theirs is expressed in terms of a different basis. 


\section{THE HOOK CASE}

Assume that $\lambda$ is a partition of $n$, and let $\mathscr{F} \lambda$ denote the set of standard Young tableaux of shape $\lambda$. For any $T \in \mathscr{F}^{\lambda}$, let $D(T)$ denote the corresponding descent set; i.e., the subset of $\{1, \ldots, n-1\}$ with $i \in D(T)$ if and only if $i$ appears in a row strictly higher than $i+1$ in $T$ (cf. Example 2.2). For each $r \geq 0$ and $T \in \mathscr{F}^{\lambda}$, we define

$$
\alpha_{r}(T)=\sum_{1 \leq k<r, k \in D(T)} k, \quad \beta_{r}(T)=\sum_{r \leq k<n, k \notin D(T)} n-k .
$$

Theorem 2.1. We have

$$
K_{\lambda, r 1^{n-r}}(q, t)=\sum_{T \in \mathscr{F} \lambda} q^{\alpha_{r}(T)} t^{\beta_{r}(T)} .
$$

Example 2.2. Take $\lambda=221, n=5, r=3$. The standard tableaux of shape 221 are

$$
\begin{array}{llllllllll}
1 & 2 & 1 & 2 & 1 & 3 & 1 & 3 & 1 & 4 \\
3 & 4, & 3 & 5, & 2 & 4, & 2 & 5, & 2 & 5 . \\
5 & & 4 & & 5 & & 4 & & 3 &
\end{array}
$$

The corresponding descent sets are

$$
\{2,4\},\{2,3\}, \quad\{1,3,4\}, \quad\{1,3\}, \quad\{1,2,4\},
$$

so we obtain

$$
K_{221,311}(q, t)=q^{2} t^{2}+q^{2} t+q+q t+q^{3} t^{2} .
$$

Proof. By [M1, Example III.4.2], we have

$$
\prod_{i \geq 1}\left(1+x_{i}\right)=\sum_{\lambda} s_{\lambda^{\prime}}\left(1, t, t^{2}, \ldots\right) S_{\lambda} .
$$

Therefore, since $J_{1^{n}}(q, t)=Q_{1^{n}}(t)=(t ; t)_{n} e_{n}$, it follows that

$$
J_{1^{n}}(q, t)=(t ; t)_{n} \sum_{|\lambda|=n} s_{\lambda^{\prime}}\left(1, t, t^{2}, \ldots\right) S_{\lambda} .
$$

By duality we therefore have

$$
J_{n}(q, t)=(q ; q)_{n} \sum_{|\lambda|=n} s_{\lambda}\left(1, q, q^{2}, \ldots\right) S_{\lambda} .
$$

Now let $c_{\mu \nu}^{\lambda}$ denote the coefficient of $S_{\lambda}$ in $S_{\mu} S_{\nu}$. Since this is also the coefficient of $s_{\nu^{\prime}}$ in the skew Schur function $s_{\lambda^{\prime}} / \mu^{\prime}$, it follows that

$$
\begin{aligned}
J_{r} \cdot J_{1^{n-r}}= & (q ; q)_{r}(t ; t)_{n-r} \\
& \times \sum_{|\mu|=r,|\nu|=n-r} s_{\mu}\left(1, q, q^{2}, \ldots\right) s_{\nu^{\prime}}\left(1, t, t^{2}, \ldots\right) \sum_{\lambda} c_{\mu \nu}^{\lambda} S_{\lambda} \\
= & (q ; q)_{r}(t ; t)_{n-r} \sum_{|\mu|=r,|\lambda|=n} s_{\mu}\left(1, q, q^{2}, \ldots\right) s_{\lambda^{\prime} / \mu^{\prime}}\left(1, t, t^{2}, \ldots\right) S_{\lambda} .
\end{aligned}
$$

It is well known (e.g., [M1, Example I.4.15]) that

$$
s_{\lambda}\left(1, q, q^{2}, \ldots\right)=\frac{1}{(q ; q)_{n}} \sum_{T \in F^{\lambda}} q^{\alpha_{n}(T)},
$$


and this easily generalizes to skew shapes; i.e.,

$$
s_{\lambda / \mu}\left(1, q, q^{2}, \ldots\right)=\frac{1}{(q ; q)_{s}} \sum_{T \in \mathscr{F}^{\lambda / \mu}} q^{\alpha_{s}(T)},
$$

where $s=|\lambda|-|\mu|$.

For any $T \in \mathscr{F} \lambda / \mu$, let $T^{\prime}$ denote the tableau obtained by transposing rows and columns and let $T^{*}$ denote the tableau obtained by rotating $T$ by $180^{\circ}$ and then substituting $i \mapsto s+1-i$. Also let $(\lambda / \mu)^{*}$ denote the skew shape obtained by rotating $\lambda / \mu$ by $180^{\circ}$. Since $i \in D(T)$ if and only if $s-i \in D\left(T^{*}\right)$, it follows that $\alpha_{s}\left(T^{*}\right)=\beta_{1}\left(T^{\prime}\right)$. Furthermore, since $s_{\lambda / \mu}=s_{(\lambda / \mu)^{*}}$, it follows that (2.2) implies

$$
\sum_{T \in \mathscr{F}^{\lambda / \mu}} t^{\alpha_{s}(T)}=\sum_{T \in \mathscr{F}^{\lambda / \mu}} t^{\beta_{1}\left(T^{\prime}\right)}
$$

Thus, by (2.1), we have

$$
\begin{aligned}
J_{r} \cdot J_{1^{s}} & =\sum_{|\mu|=r, T_{1} \in \mathscr{F}^{\mu}} q^{\alpha_{r}\left(T_{1}\right)} \sum_{|\lambda|=n, T_{2} \in \mathscr{F}^{\lambda / \mu}} t^{\beta_{1}\left(T_{2}\right)} S_{\lambda} \\
& =\sum_{|\lambda|=n, T \in \mathscr{F}^{\lambda}} q^{\alpha_{r}(T)} t^{\beta_{r+1}(T)} S_{\lambda} .
\end{aligned}
$$

On the other hand, we claim that

$$
J_{r} \cdot J_{1^{s}}=\frac{1-t^{s}}{1-q^{r} t^{s}} J_{\left(r+1,1^{s-1}\right)}+\frac{1-q^{r}}{1-q^{r} t^{s}} J_{\left(r, 1^{s}\right)} .
$$

To prove this, note that by [M2, (4.8)] one knows that the $J_{\lambda}$-expansion of $J_{r} \cdot J_{\mu}$ only involves those partitions $\lambda$ such that $\lambda / \mu$ is a horizontal $r$-strip. Hence, there exist scalars $c_{1}, c_{2} \in \mathbf{Q}(q, t)$ such that

$$
J_{r} \cdot J_{1^{s}}=c_{1} J_{\left(r+1,1^{s-1}\right)}+c_{2} J_{\left(r, 1^{s}\right)} .
$$

These scalars can be computed directly from [M2, (5.12)], but it is even easier to apply the specialization $\varepsilon_{a, t}$ (cf. (1.6)), yielding

$$
(1-a)=c_{1}\left(1-q^{r} a\right)+c_{2}\left(t^{s}-a\right)
$$

after cancellation of common factors. By choosing $a=t^{s}$ and $a=q^{-r}$, we obtain $c_{1}=\left(1-t^{s}\right) /\left(1-q^{r} t^{s}\right)$ and $c_{2}=\left(1-q^{r}\right) /\left(1-q^{r} t^{s}\right)$, thus proving (2.4).

Finally, combining (2.3) and (2.4), we obtain

$$
\sum_{T \in \mathscr{F}^{\lambda}} q^{\alpha_{r}(T)} t^{\beta_{r+1}(T)}=\frac{1-t^{s}}{1-q^{r} t^{s}} K_{\lambda,\left(r+1,1^{s-1}\right)}(q, t)+\frac{1-q^{r}}{1-q^{r} t^{s}} K_{\lambda,\left(r, 1^{s}\right)}(q, t) .
$$

As $r$ ranges from 0 to $n$ (with $s=n-r$ ), this yields a sequence of identities that uniquely determine all of the coefficients $K_{\lambda, r 1^{n-r}}(q, t)$. Therefore, it suffices to show that

$$
\left(1-q^{r} t^{S}\right) q^{\alpha_{r}(T)} t^{\beta_{r+1}(T)}=\left(1-t^{S}\right) q^{\alpha_{r+1}(T)} t^{\beta_{r+1}(T)}+\left(1-q^{r}\right) q^{\alpha_{r}(T)} t^{\beta_{r}(T)}
$$

for all $T \in \mathscr{F}^{\lambda}$.

For this, observe that if $r \in D(T)$, then $\alpha_{r+1}(T)=r+\alpha_{r}(T)$ and $\beta_{r+1}(T)=$ $\beta_{r}(T)$; similarly, if $r \notin D(T)$, then we have $\alpha_{r+1}(T)=\alpha_{r}(T)$ and $\beta_{r}(T)$ 
$=s+\beta_{r+1}(T)$. Hence, the verification of (2.5) reduces to the trivialities

$$
\begin{aligned}
& \left(1-q^{r} t^{s}\right)=q^{r}\left(1-t^{s}\right)+\left(1-q^{r}\right), \\
& \left(1-q^{r} t^{s}\right)=\left(1-t^{s}\right)+t^{s}\left(1-q^{r}\right) .
\end{aligned}
$$

\section{REFERENCES}

[A] G. E. Andrews, The theory of partitions, Addison-Wesley, Reading, MA, 1976.

[GH] A. M. Garsia and M. Haiman, A graded representation model for Macdonald's polynomials, Proc. Nat. Acad. Sci. U.S.A. 90 (1993), 3607-3610.

[JJ] N. H. Jing and T. Józefiak, A formula for two-row Macdonald functions, Duke Math. J. 67 (1992), 377-385.

[M1] I. G. Macdonald, Symmetric functions and Hall polynomials, Oxford Univ. Press, Oxford, 1979.

[M2] — A new class of symmetric functions, Publ. I.R.M.A. Strasbourg, Actes $20^{e}$ Séminaire Lotharingien, 1988, pp. 131-171.

Department of Mathematics, University of Michigan, Ann Arbor, Michigan 481091003 